\title{
La perspectiva de la efectividad escolar para la evaluación de la calidad de la educación
}

GUADALUPE RUIZ CUÉLLAR

Departamento de Educacion/UAA

\section{INTRODUCCIÓN}

D

urante muchos años la investigación educativa reforzó el supuesto de que las posibilidades de logro académico de los alumnos estaban fuertemente determinadas por las condiciones socioeconómicas y culturales de sus medios de procedencia, condiciones externas al sistema educativo. Esta conclusión dejaba poco qué hacer a la escuela, pues Iimitaba seriamente sus potencialidades para influir positivamente sobre el éxito académico de sus alumnos y éste -o su contraparte, el fracaso escolar-, debían ser explicados por la influencia de factores genéticos o factores asociados con la clase social de los alumnos y el medio en que se desenvolvían. Sin embargo:

... en años recientes, y debido fundamentalmente a un importante viraje en la investigación educativa que diversificó sus metodologías, volvió sus ojos

1. En el conjunto de los esfuerzos de muy diversa naturaleza y alcance que se vienen realizando en el estado de Aguascalientes en materia de evaluación de la calidad de la educación, hemos desarrollado un proyecto de investigación que centra su interés en la identificación de los niveles en que se logran los aprendizajes establecidos por el curriculum de la educación primaria y en la determinación de los factores escolares que influyen sobre esos resultados de aprendizaje. Este artículo ofrece una síntesis de algunos de los referentes teóricos que guiaron el desarrollo de la investigación. 
a la escuela y al sistema, cambió sus métodos, y se preguntó sobre las variaciones en las características de la oferta y su papel en la explicación de la desigualdad educativa, hemos podido constatar que ...., el operar del propio sistema educativo y de la escuela tienen un peso independiente de no poca importancia en la explicación de la desigualdad. Con ello se cuestiona el anterior escepticismo, y se comienza a considerar que en efecto, hay algo que se puede hacer desde el sistema educativo para comenzar a desvincular educación con desigualdad. ${ }^{2}$

Sin desconocer que el aprendizaje de los alumnos es producto de la interacción compleja de variables extraescolares y escolares, la aportación que puede hacer la escuela al logro académico de los alumnos y, en un sentido más amplio, al mejoramiento de la calidad de la educación es hoy en día ampliamente reconocido: las variables escolares "tienen su propio peso" que no se reduce al de las extraescolares y que significa para los educadores, "un margen significativo de acción, con posibilidades ... de incidir realmente en los resultados, más allá de los condicionamientos del entorno, si no son excesivamente desfavorables". ${ }^{3}$

La calidad de la educación es un concepto que no se presta fácilmente a definición. Existen planteamientos diversos sobre lo que debe entenderse por ésta e incluso hay quienes sostienen que es imposible definirla con rigor y precisión. Con todo, si se pretende juzgar la calidad de un sistema educativo o dé alguno de sus componentes, es necesario contar con una concepción de lo que constituye la calidad, aún reconociendo que puede no ser satisfactoria para todos. En particular, nos parece que una forma orientadora de concebir la calidad educativa es la que considera que se trata de un concepto que comprende los siguientes aspectos:

2. Sylvia Schmelkes, "Desigualdad social y educación". Ponencia presentada en el Foro de Consulta Popular sobre Justicia Educativa para el Plan Nacional de Desarrollo 1995-2000, Pachuca, Hidalgo, abril 1995.

3. Felipe Martínez, La Calidad de la Educación en Aguascalientes. Diseño de un Sistema de Monitoreo, México, UAA-IEA, 1996. 
Relevancia: los objetivos y contenidos educativos deben ser evaluados en función de su aportación para la vida de los educandos. Eficacia: suponiendo que los objetivos y contenidos sean relevantes, la educación será tanto más eficaz cuanto mayor sea el número de alumnos que alcance un dominio adecuado de tales objetivos y contenidos; es fácil ver que este concepto de eficacia comprende los de cobertura educacional (acceso y permanencia) y nivel de aprendizaje (logro de objetivos), y que está estrechamente relacionado con el siguiente: Equidad: se trata de la característica del sistema educativo consistente en atender diferenciadamente a sus alumnos, ofreciéndoles los elementos necesarios según las características individuales y del entorno social de cada uno, para dar a todos las máximas oportunidades de alcanzar las metas de aprendizaje relevante que correspondan... Eficiencia: ... se refiere a la relación entre los resultados obtenidos por la educación y los insumos requeridos para ello. ${ }^{4}$

Sin restar importancia al conjunto de aspectos o dimensiones de la calidad educativa mencionadas, lo cierto es que en la tradición evaluativa que ha imperado no sólo en nuestro país, sino en otros a lo largo de varias décadas, la evaluación del aprovechamiento escolar ha ocupado un lugar central. ${ }^{5}$ En términos del concepto de calidad que hemos presentado, esto tiene relación con la dimensión de eficacia.

En lo que a nosotros compete, el énfasis sobre la medición y evaluación de los niveles de aprendizaje logrados por los escolares, sobre

4. Este concepto de calidad es producto de un trabajo colegiado dirigido por Pablo Latapí y se encuentra contenido en la Propuesta de Reforma de la Educación (presentada ante el C. Secretario de Educación Pública, Lic. Manuel Bartlett Diaz, Mecanoescrito, 1989. Una versión más refinada del concepto aparece en la obra ya citada de Felipe Martínez.

5. Diversos estudios evidencian una comprensión de la calidad de la educación centrada en los aprendizajes. Por ejemplo, Eduardo Vélez, Ernesto Schiefelbein y Jorge Valenzuela, Factores que afectan el rendimiento académico en la educación primaria (Revisión de la literatura para América Latina), Washington, Banco Mundial, s/f., señalan que en el contexto de su trabajo, usan el términe "logro educativo", como una medida de la calidad de la educación. Algo similar se observa en la propuesta elaborada por UNESCO/OREALC, Medición de la calidad de la educación básica: ¿jpor qué, cómo y para qué? Una propuesta sobre el mejoramiento de la calidad y las nuevas demandas de información, Santiago, REPLAD, 1992. 
la eficacia de un sistema educativo y de sus instituciones educativas singulares, las escuelas, nos ha llevado a reconocer un importante terreno de investigación, el de la efectividad escolar.

La efectividad escolar se relaciona específicamente con los logros en aprendizajes:

Si uno empieza con la definición básica de "efectivo" -producir un resultado o producto deseado- y usa esto para definir una escuela efectiva, rápidamente se da cuenta que existe un consenso virtual, al menos conceptualmente, sobre lo que constituye una escuela efectiva. En una forma u otra, una escuela efectiva usualmente es descrita como una donde todos los estudiantes logran con maestría el currículum pretendido. En otros términos, en la evolución del discurso sobre escuelas efectivas se plantean dos cuestiones básicas: ¿efectivas en qué? y ¿efectivas para quiénes? ... "Efectivas en qué" requiere que la enseñanza sea tal que los estudiantes logren con maestría el currículum establecido. "Efectivas para quiénes" significa todos los estudiantes. ${ }^{6}$

De acuerdo a este planteamiento, lograr que todos los estudiantes de una escuela dominen el currículum establecido es lo que define o caracteriza a una escuela efectiva. Rápidamente se puede observar sin embargo, que ésta es una definición cuestionable de la efectividad pues por factores que pueden ser de índole propiamente escolar, socioeconómica y cultural o individual, no parece realista pensar que todos los estudiantes logren el mismo nivel de aprendizaje o dominio de los contenidos establecidos por un currículum. Por ello, una definición más operativa es la que propone que las escuelas efectivas son aquéllas que son capaces de promover el aprovechamiento académico promedio de los estudiantes a los que atienden. ${ }^{7}$ Desde esta perspectiva, controlando las variaciones individuales al interior de cada centro es-

6. Daniel Levine y Lawrence Lezzote, Unusually effective schools: a review and analysis of research and practice. National Center for Effective Schools Research and Development, Madison WI, 1990.

7. Thomas Good y Jeremy Brophy, "School Effects", en: Merlin Wittrock (ed), Handbook of Research on Teaching, Third Edition, Nueva York, McMillan Publishing Company, 1986. 
colar a través de un indicador sintético como lo es el promedio, es posible identificar diferentes grados de efectividad en las escuelas.

Pero, ¿qué es lo que hace a una escuela capaz de promover el aprovechamiento académico promedio de sus estudiantes? ¿Qué es lo que la distingue como escuela efectiva?

La idea de la efectividad escolar remite justamente a esa capacidad ahora ampliamente reconocida de que la escuela tiene un peso específico importante en la generación de los resultados de aprendizaje, aún en condiciones contextuales adversas. Este reconocimiento se ha manifestado básicamente en dos formas, por un lado, como un movimiento en pro de la mejoría de las escuelas; por otro, como un terreno de investigación en constante expansión que ha contribuido a clarificar los mecanismos a través de los cuales las escuelas pueden ser efectivas al incidir positivamente sobre el éxito académico de sus estudiantes.

Desde esta segunda perspectiva, cabría señalar en primera instancia que muchas de las primeras investigaciones sobre las escuelas efectivas, identificaron un conjunto básico de características o que distinguen a las escuelas de acuerdo a su grado de efectividad. En 1987, Edmonds planteó las siguientes: énfasis sobre la adquisición de habilidades básicas por parte de los estudiantes; altas expectativas respecto a los estudiantes; fuerte liderazgo administrativo; monitoreo frecuente del progreso de los estudiantes y, clima ordenado y propicio para el aprendizaje. ${ }^{8}$

La mayor parte de la investigación reciente ha utilizado esos cinco o siete correlatos para distinguir entre escuelas más y menos efectivas; sus resultados en general, han continuado apoyando las conclusiones de esos estudios. Con todo, la gama de factores identificados hoy en día, se ha diversificado notablemente hasta incluir un conjunto muy amplio de correlatos de la efectividad escolar.

En lo que resta del texto se presenta un modelo de factores que trata de reflejar las principales líneas de investigación y hallazgos pertinen-

8. En Levine y Lezotte, Op. Cit. 
tes al concepto de efectividad escolar. Daremos cuenta fundamentalmente de aquellos que se manifiestan a nivel de la institución escolar; dado que las variables de la efectividad de la enseñanza, es decir, las que tienen expresión al nivel del aula, constituyen de suyo un campo extenso y complejo de investigación, hemos preferido no abordarlo en este escrito, más que de manera marginal y en la medida en que ciertos factores de la enseñanza efectiva se relacionan con otros de orden institucional. El modelo consta de un conjunto de grandes categorías que incluyen en la mayoría de los casos, varios factores específicos.

\section{Clima y cultura escolar}

\section{Ambiente ordenado}

Un "ambiente seguro, ordenado" conductivo a la enseñanza y el aprendizaje y aspectos similares del clima escolar, frecuentemente han sido señalados como una característica importante de las escuelas efectivas. Es claro que el aprendizaje ocurre más fácilmente si el orden prevalece, es decir, si estudiantes y maestros asisten a clases regularmente y de acuerdo a un tiempo establecido, si las instalaciones están limpias y en buen estado, y si los materiales necesarios se proporcionan en forma rutinaria. Además de estas condiciones básicas del funcionamiento escolar, se identifican también ciertas características de los procesos en el aula -el manejo efectivo del aula-, que muestran la existencia de un ambiente escolar ordenado, favorable al aprendizaje: los maestros logran y mantienen la atención de sus estudiantes; mantienen también la disciplina; empiezan y terminan las lecciones a tiempo; permiten que los estudiantes conozcan lo que se espera de ellos, y monitorean y evalúan el desempeño de los alumnos. ${ }^{9}$

9. Marlaine Lockheed y Adriaan Verspoor, Improving Primary Education in Developing Countries, Nueva York, Oxford University Press, 1991. 
Compromiso del personal docente en torno

a una misión compartida y articulada, enfocada a mejorar el aprendizaje

La investigación sobre escuelas efectivas indica que el compromiso del personal con la tarea de ayudar a todos los estudiantes a dominar con maestría objetivos importantes de aprendizaje, es una cuestión clave para diferenciar entre escuelas más y menos efectivas. Las escuelas que se desempeñan mejor, se caracterizan por la aceptación de los maestros de su responsabilidad para el aprovechamiento de los estudiantes; además, esta actitud se manifiesta en una variedad de acciones que involucran un compromiso inusual de su tiempo y energía en ayudar al éxito de los estudiantes.

Ahora bien, sin lugar a dudas, la mayoría de las escuelas tienen al menos algunos maestros que están fuertemente comprometidos a ayudar a sus estudiantes a aprender y que actúan en concordancia con esta creencia en la práctica. Pero, en este sentido, lo que distingue a las escuelas efectivas de otras menos efectivas con grupos similares de estudiantes, es la aceptación convencida por parte de todo su personal, de este compromiso.

Orientación a la resolución de problemas

En estrecha relación con las normas culturales que enfatizan la mejoría del aprovechamiento de todos los estudiantes, tanto el personal directivo como el docente de las escuelas efectivas muestran una actitud que podría sintetizarse en la siguiente expresión: "si lo que nosotros estamos haciendo no muestra que estemos trabajando para todos los estudiantes, particularmente los de bajo rendimiento, podríamos identificar los obstáculos que enfrentamos y tratar de hacer algo para abarcar a todos".

En este sentido se entiende la "orientación hacia la resolución de problemas", como esa disposición a identificar las debilidades de las 
prácticas existentes y cambiarlas si es necesario, a fin de implementar enfoques más efectivos.

Cohesión del personal, colaboración, consenso, comunicación y "colegialidad"

Casi por definición, los profesores y directivos comprometidos con una misión escolar enfocada a la mejoría académica de todos los estudiantes, tienden a mostrar gran cohesión y consenso con respecto a las metas que de ahí se derivan.

Como lo han establecido los extensos análisis de Purkey y Smith (1983), Fuller e Izu (1986), Rosenholtz (1985), Cohen (1988), y otros $^{10}$, la cohesión y el consenso son particularmente importantes en organizaciones, tales como las escuelas, en las que el colectivo docente debe alcanzar una variedad de metas difíciles y a veces conflictivas, donde los medios para realizarlas no son siempre claros, y donde factores tanto externos como internos, tienden a reducir la claridad de las metas y a fragmentar los esfuerzos de mejoría. Muchos analistas también creen que la "colegialidad" es importante en mejorar la cohesión y las comunicaciones, la identificación y resolución de problemas, y en reforzar otros aspectos de la efectividad.

\section{Involucramiento del personal en la toma de decisiones}

Dada la evidencia ya mencionada en el sentido de que las escuelas efectivas muestran puntuaciones altas en los patrones de comunicación de su personal y una orientación hacia la resolución de problemas prioritarios, no es sorprendente que numerosos investigadores hayan identificado que tales escuelas alcanzan puntajes altos

10. Citados por Levine y Lezotte, Op. Cit. 
también, en lo que se refiere a tomar en cuenta a los profesores en la toma de decisiones.

Ahora bien, no es claro aún si el hecho de involucrar al personal docente siempre o usualmente, ha sido una consideración crítica en la generación de la efectividad, ni es claro tampoco si este aspecto ha sido más valioso cuando se define restrictivamente en términos de solicitar sugerencias, o bien, cuando se define más ampliamente, en términos de una participación más completa en la toma de decisiones. Muy probablemente, la forma apropiada para introducir este elemento y la extensión en la cual debe ser utilizado, depende fuertemente de consideraciones que involucran las personalidades y experiencias de maestros y directivos, los tipos y urgencia de los problemas que encuentran, la disponibilidad de asistencia externa para el desarrollo organizacional y muchos otros factores. Esta clase de consideraciones muestra de manera muy clara que más allá de las variables ya identificadas como características de las escuelas efectivas, hay todavía más elementos que, relacionados con esas variables, definen patrones específicos de efectividad.

Énfasis de la escuela en el reconocimiento

del desempeño positivo

Algunos académicos que han revisado la investigación sobre escuelas efectivas, han concluido que las escuelas efectivas sistemática y públicamente reconocen a los estudiantes que son exitosos académicamente y/o demuestran otras conductas positivas. Por ejemplo, Purkey y Smith (1983)" examinan la investigación sobre cultura escolar y concluyen que una de las características culturales que contribuyen a la efectividad es "el reconocimiento escolar del

11. Citados por Levine y Lezotte, Op. Cit. 
éxito académico: honrar públicamente el rendimiento académico y recalcar su importancia alienta a los estudiantes a adoptar normas y valores similares".

Este factor se encuentra obviamente relacionado con las expectativas sobre el desempeño de los estudiantes, el liderazgo y el énfasis sobre el aprendizaje. Es importante señalar que según lo muestran algunos estudios, hay escuelas efectivas que han extendido esta característica más allá de la esfera académica, reconociendo y recompensando a los alumnos por la asistencia o conductas cívicas positivas. Destacamos este hallazgo porque a nuestro parecer, muestra cómo aún cuando la efectividad tiende a entenderse centralmente referida a aprendizajes de orden cognitivo, no deja de prestarse atención también, a otros aspectos del desempeño de los estudiantes, sobre los que también se espera que incida la escuela.

\section{LIDERAZGO SOBRESALIENTE}

El director de una escuela es -como lo muestra la gran mayoría de los estudios-, el componente más crítico del liderazgo, en una escuela eficaz. Toca a él establecer o proponer al cuerpo de maestros la misión y las metas de la escuela; promover una instrucción de calidad; impulsar un clima de aprendizaje académico, estableciendo expectativas y estándares positivos; desarrollar un buen ambiente de trabajo proporcionando oportunidades a los estudiantes de involucrarse en actividades escolares significativas, desarrollando la colaboración y cohesión de la planta docente, consiguiendo recursos externos para apoyar el logro de las metas de la escuela y estableciendo relaciones entre el hogar y la escuela, particularmente respecto a la misión primaria de educar a los estudiantes.

Algunos factores que destaca particularmente el modelo de Levine y Lezotte son los siguientes. 
Énfasis en la selección y reemplazo

de maestros tendiente a la efectividad

La investigación sobre los directores de escuelas efectivas establece claramente que ellos están mucho más inclinados que el director promedio a asumir un importante papel en la selección de los maestros que trabajarán en su institución, así como en la transferencia de aquéllos a quienes consideren que no contribuyen a la efectividad de la escuela.

En muchos casos, esta función de liderazgo ha requerido inversiones de gran cantidad de tiempo y energía de parte del director, así como buena voluntad para hacer frente a las prácticas de asignación y remoción de profesores establecidas por las autoridades. En este sentido, es frecuente que los directores de escuelas efectivas entren en conflicto o manifiesten desacuerdo con los criterios centrales de selección y reemplazo de profesores.

El énfasis en la selección y remoción de maestros es más evidente durante los primeros años cuando una escuela se está moviendo hacia la efectividad. Después de que los directores han registrado un éxito significativo en la constitución y/o motivación de un conjunto de profesores capaz de trabajar colegiadamente para mejorar su efectividad, el cambio se reduce y la composición del grupo se estabiliza. Dado que los maestros y los estudiantes experimentan mucho más éxito en esta situación, es más fácil reclutar y retener profesores capaces.

Monitoreo frecuente y personal de las actividades de la escuela

En relación con la sólida conclusión de que el director es usualmente un factor clave para que en una escuela se manifiesten conductas de liderazgo asociadas con la efectividad escolar, hay que mencionar el hallazgo -también fuertemente apoyado por la evidencia empírica- de que uno de los aspectos de esas conductas se 
refiere a la realización de visitas frecuentes a los salones de clase combinadas con una constante vigilancia personal de las actividades que ocurren en la escuela.

Ahora bien, Levine y Lezotte advierten que este aspecto del liderazgo en escuelas efectivas no necesariamente supone procedimientos de supervisión clínica, sistemática que requerirían a los directores invertir grandes cantidades de tiempo, observaciones formalmente estructuradas y pre y post-entrevistas con sus maestros; sobre todo, si ello implica distraerlos de otras cuestiones más importantes.

Más bien, lo que indica la literatura es que los directores de estas escuelas acuden frecuentemente a los salones de clase y a otros espacios de la escuela, asimismo que conversan informalmente con sus profesores de manera regular. Este patrón de visitas al aula impredecibles y conversaciones ejemplifica lo que se ha denominado "management by walking around". 12

\section{Alta inversión de tiempo y energía en acciones de mejoría de la escuela}

Producir y mantener mejorías duraderas en el aprovechamiento es una tarea difícil y compleja que requiere generalmente la inversión de mucho tiempo y energía de parte del director y de otros líderes de la escuela. A pesar de que presumiblemente se trata de una variable de importancia, se ha investigado poco dado que es difícil obtener datos válidos, a menos que se realicen costosas observaciones que

12. Una traducción más o menos literal diría "administración por medio de contacto directo informal"; lo que se trata de resaltar es que en las escuelas efectivas el director cumple su función no desde el escritorio, sino a través de una interacción directa con las personas que integran el centro escolar, "paseando" o acudiendo a sus diversos sitios de trabajo. Peters y Waterman (1982), citados por Sam Stringfield, "A model of elementary school effects", Advances in school effectiveness research and practice, Gran Bretaña, Pergamon, 1994. 
evalúen las conductas de liderazgo sobre periodos relativamente largos de tiempo. En todo caso, no habría que desconocer que seguramente hay disminuciones y flujos en los patrones de inversión de energía y tiempo por parte de los directores en función de variables contextuales.

Por otro lado, "hasta ahora no es muy claro, y quizá nunca lo sea, si dedicar grandes cantidades de tiempo y energía es más importante como un prerrequisito para enfrentar las difíciles tareas de mejoría de las escuelas, o como un medio para comunicar y asegurar un compromiso de la institución, o si es igualmente importante para ambos propósitos". ${ }^{13}$

\section{Apoyo a los maestros}

Los directores de escuelas efectivas destinan gran cantidad de tiempo a apoyar a sus profesores. Muchas de las funciones de apoyo de las que el líder es al menos en parte responsable, incluyen la provisión de recursos, la asistencia técnica y la capacitación a profesores. Ello sin duda se relaciona con cuestiones como: su orientación a la resolución de problemas o su inversión de tiempo en la escuela. A su vez, con muy pocas excepciones, los directores requieren asistencia de una o más personas que les apoyen en cuestiones instruccionales. El hecho de contar con personas que apoyen al director -y a los profesores- (por ejemplo, especialistas en diversas áreas del currículum), incide positivamente en la mejoría de los aprendizajes de los alumnos.

Por lo que toca a la capacitación a profesores, según los hallazgos de las investigaciones que reportan este factor, en las escuelas efectivas tienden a privilegiarse las acciones de entrenamiento que tienen lugar en la propia escuela. Stedman (1987) ha proporciona-

13. Levine y Lezotte, Op Cit. 
do una excelente descripción con ejemplos, de este énfasis en el desarrollo práctico del personal en algunas de esas escuelas:

... la mayoría, usan procedimientos prácticos de entrenamiento en el mismo sitio de trabajo, los cuales han sido ajustados a las necesidades específicas de los miembros del personal y los estudiantes. Las escuelas efectivas dan demostraciones de lecciones a los maestros inexpertos... proporcionan periodos extra de preparación para los novicios... (permiten que ellos observen) a maestros experimentados... se filma el desempeño de los maestros para ayudar a mejorar la instrucción y las evaluaciones... y ayudan a los maestros a seleccionar materiales y técnicas de enseñanza... El énfasis a través de todo el entrenamiento estuvo puesto en intercambiar técnicas prácticas de enseñanza y en hacer del entrenamiento una parte integral de un ambiente educacional colaboracionista. ${ }^{14}$

Como una expresión de su liderazgo académico y administrativo, el director de una escuela efectiva impulsa este tipo de procesos de capacitación, entrenamiento y a fin de cuentas, comunicación de una cultura común a sus profesores.

\section{OTRAS CATEGORÍAS DE LA EFECTIVIDAD ESCOLAR}

Las dos categorías presentadas en las páginas precedentes constituyen las más complejas dado que incluye cada una de ellas, un número más o menos amplio de factores. En este apartado agrupamos aquéllas que se descomponen en un número menor de elementos. Todas ellas son sin duda, también importantes en la generación y explicación de diferentes niveles de efectividad escolar.

Como ya se ha dicho, uno de los primeros hallazgos de la investigación en este terreno es el que se refiere a la existencia de altas expectativas respecto al logro de los estudiantes en aquellas escuelas más efectivas; esto se ha observado tanto en estudios de caso

14. Citado por Levine y Lezotte, Op Cit. 
como en estudios con muestras que incluyen diferentes grados o niveles de efectividad escolar:

Las escuelas efectivas establecen claramente metas definidas para el aprovechamiento académico, y concentran los recursos de que disponen y sus actividades en obtenerlas. En las escuelas elementales, para que los estudiantes adquieran tanto habilidades básicas como habilidades complejas, las escuelas deben focalizar su currículum hacia la enseñanza de esas habilidades, proporcionar tiempo suficiente para su enseñanza, y coordinar la instrucción a través de los diferentes grados. Las escuelas efectivas continuamente monitorean el progreso de sus estudiantes para determinar si las metas instruccionales están siendo conseguidas... también tienen altas expectativas respecto al trabajo y el aprovechamiento. El concepto de la escuela como un lugar de aprendizaje es comunicado claramente a los estudiantes, y se espera un compromiso para el aprendizaje en cada salón de clases. Las expectativas se manifiestan en los estándares de desempeño fijados por la escuela -bajos estándares reflejan expectativas pobres, altos estándares reflejan expectativas elevadas. ${ }^{15}$

Aparecen en la cita precedente varios elementos más, que es importante destacar y que muestran cómo los factores de la efectividad se correlacionan entre sí; por ejemplo, el desarrollo de expectativas altas va aparejado al monitoreo del progreso de los estudiantes, el cual no sólo es realizado para obtener datos de diagnóstico $0^{16}$, sino también, para ayudar a comunicar las expectativas que se tienen y una fuerte presión académica; la disciplina es firme en parte porque un clima disruptivo haría ver a las altas expectativas como poco realistas.

A su vez, el logro de las metas que la institución se fija en términos del aprendizaje de los escolares a los que atiende, exige que todas las acciones de enseñanza estén encaminadas en esa dirección. En este sentido, la optimización del tiempo destinado al proceso de

15. Lockheed y Verspoor, Op. Cit.

16. Lo cual tiene relación con la orientación hacia la resolución de problemas que caracteriza a las escuelas efectivas. 
enseñanza-aprendizaje es una variable crucial en las escuelas efectivas; en ellas, la planta docente se asegura de proporcionar a los estudiantes el tiempo necesario para el aprendizaje.

Así, varias investigaciones han descrito escuelas efectivas en las que se invierte relativamente poco tiempo en las transiciones entre clases, recesos u otras actividades extrañas al aprendizaje. Si bien, la mayoría de estos estudios describen escuelas efectivas de bajo nivel socioeconómico en las que el tiempo para el aprendizaje resulta ser de particular importancia porque los estudiantes requieren de mucho apoyo instruccional, hay también resultados de investigación que muestran que aún en escuelas con condiciones socioeconómicas más favorables, aquéllas que logran mejores niveles de efectividad, prestan especial atención a la maximización del tiempo de aprendizaje.

Una importante esfera de factores de la efectividad escolar tiene expresión al nivel del aula; es claro que existe una relación lógica entre los campos de la institución escolar en su conjunto y las aulas individuales. Sin embargo, lo cierto es que en términos de investigación, durante los pasados 20 o 25 años las áreas de "efectividad docente" y "efectividad escolar" han emergido separadamente; varias son las razones que explicarían el desarrollo independiente de estos dos campos de estudio, aunque no pretendemos abordarlas en este escrito. ${ }^{17}$ En todo caso, sí parece importante destacar que un modelo más exhaustivo de la efectividad escolar debe incluir no sólo variables relativas al nivel de la escuela -como las que aquí hemos destacado-, sino también, relacionadas con los procesos que ocurren al interior de sus aulas, en la interacción entre maestros y estudiantes.

La efectividad en la enseñanza -decíamos ya-, como campo de investigación ofrece al igual que el de la efectividad escolar, innu-

17. Una explicación detallada puede verse en: Charles Teddlie, "The integration of classroom and school process data in school effectiveness research", Advances in school ... 
merables hallazgos. Cabría agregar tan sólo a manera de ejemplo, el modelo de instrucción efectiva propuesto por Slavin ${ }^{18}$, que enfatiza los elementos sobre los que maestros y escuelas pueden actuar directamente:

1. Calidad de la instrucción: el grado en el cual la información o las habilidades son presentadas de forma que los estudiantes puedan aprenderlas fácilmente. La calidad de la instrucción es en gran medida producto de la calidad del currículum y de la presentación misma de la lección.

2. Niveles apropiados de instrucción: el grado en el cual el maestro está seguro de que los estudiantes están listos para aprender una nueva lección. El nivel de instrucción es apropiado cuando una lección ni es demasiado fácil ni demasiado difícil para los estudiantes.

3. Incentivos: el grado en el cual el maestro se asegura que los estudiantes están motivados para trabajar y aprender el material que será presentado.

4. Tiempo: el grado en el cual los estudiantes disponen del tiempo suficiente para aprender el material que les está siendo enseñado.

Los cuatro elementos de este modelo QAIT (Quality, Appropiatness, Incentive, Time) tienen una característica importante: todos deben ser adecuados para que la instrucción sea efectiva. La instrucción efectiva no es sólo buena enseñanza; no importa qué tan alta sea la calidad de la instrucción, los estudiantes no aprenderán una lección si les falta la información o las habilidades necesarias previas, si carecen de motivación o si no disponen del tiempo que necesitan para aprender una lección. En el otro extremo, si la calidad de la instrucción es baja, entonces no hará mucha diferencia qué tanto sepan los estudiantes, qué tan motivados estén o de cuánto tiempo dispongan.

18. En: Eugene Schaffer y otros, "The contributions of classroom observation to school effectiveness research", Advances in school ... 


\section{CONCLUSION}

En este artículo hemos tratado de ofrecer una muestra de los hallazgos de la investigación sobre escuelas efectivas. Conviene señalar -aunque posiblemente los lectores ya se hayan percatado de ello- que la literatura sobre el tema nos habla centralmente de lo que ocurre en escuelas de países desarrollados. Con todo y lo sugerente que este discurso pudiera resultar no sólo para los investigadores de la educación sino también para quienes intervienen directamente en los procesos educativos como docentes o directivos, un sentido de realismo se hace necesario al considerar nuestra propia situación.

Se podrá concluir que la mayoría, si no es que todos los factores de la efectividad escolar se refieren no a las características más o menos estáticas de los centros escolares (tales como su ubicación en el medio urbano o rural, si son públicas o privadas, de turno matutino o vespertino, etc.), sino a variables procesuales que requieren para manifestarse de adecuadas còndiciones estructurales, muchas de ellas definidas fuera de la esfera propia de las instituciones.

Con todo, si como hemos tratado de mostrar, las escuelas pueden hacer algo para incidir positivamente sobre el éxito académico de sus estudiantes, el conocimiento que hoy en día se tiene sobre la efectividad escolar, producto de la investigación sistemática sobre la materia, ofrece ideas sugerentes sobre una multiplicidad de ámbitos en los que se puede empezar a actuar. El terreno es fértil tanto para la investigación como para la práctica educativa misma. 중 